\title{
Constructive-geometry: the integration of generation and construction systems in a case-study
}

\section{SIGRADI2018 TECHNOPOLITICAS \\ xxii congresso da sociedade \\ 22th conference of the \\ iberoamerican society of digital graphics \\ 07|08|09|novembro|2018 \\ iau usp | são carlos | sp br}

\author{
Maria Elisa Regadas Reis Vianna \\ Universidade Federal Rio Janeiro, LAMO, PROURB | Brazil | elisarrvianna@gmail.com \\ Gonçalo Castro Henriques \\ Universidade Federal Rio Janeiro, LAMO, PROURB | Brazil | gch@fau.ufrj.br \\ Andrés Martin Passaro \\ Universidade Federal Rio Janeiro, LAMO, PROURB | Brazil | andrespassaro@fau.ufr..br
}

\begin{abstract}
This project reflects about the use of the term constructive geometry, based on the development of a case study. Even if we posses the digital tools and processes to develop a design, it is not so clear how to combine the generation and the necessary tools to materialize it. To find form we rely on algorithmic generation unfolding this term with digital fabrication, to include material and techniques feedback. Constructive geometry looks for an inclusive computational design to integrate generation and fabrication. This process is tested and documented in the development of a studentl graduation project.
\end{abstract}

Keywords: Constructive geometry, digital integration, form generation, digital fabrication

\section{INTRODUÇÃO}

Existe na academia, uma dificuldade em incorporar no processo de projeto as novas ferramentas e processos digitais. O LAMO - Laboratório de Modelos 3D e Fabricação Digital tem procurado alterar este paradigma, através de workshops, cursos de curta duração, disciplinas eletivas e no desenvolvimento de trabalhos finais de graduação.

O processo tradicional de projeto em arquitetura vigente na academia, em geral e, na brasileira em particular, ainda utiliza pouco o potencial generativo dos processos digitais. As novas tecnologias são frequentemente utilizadas apenas durante o desenvolvimento final de projeto, contribuindo menos para a sua concepção, mantendo assim uma metodologia sequencial, herdeira do modernismo, mas com novas técnicas. A forma é prédefinida utilizando processos tradicionais, sendo menos explorados os processos algoritmos para encontrar famílias de soluções (paramétricas). De maneira análoga, os processos de fabricação, apesar de mais frequentes, aparecem separados do processo de geração, sendo apenas utilizados após terminado o projeto.

Este artigo descreve o desenvolvimento de um trabalho final de graduação, que utiliza como ponto de partida a metodologia desenvolvida na disciplina Modelagem Digital em Arquitetura (MDA/ FAU-UFRJ), que procura incentivar a integração digital. Esta disciplina começa por introduzir os alunos na evolução das técnicas e processos digitais em projeto dos últimos 50 anos. Combina a introdução de referências teóricas e metodológicas oriundas de autores como Mitchell, Kolarevic, Oxman, Terzidis, Celani, Duarte, Menges
(Sobre o tema consultar Henriques, 2016). Inclui uma explicação sobre as técnicas de fabricação digital disponíveis, citando exemplos construídos. Após este enquadramento teórico-prático, são desenvolvidos um conjunto de exercícios de geração da forma. Esta integração digital, procura legitimar uma metodologia de busca da forma com as novas técnicas disponíveis durante todo processo.

A partir deste embasamento, o termo, "Geometria Construtiva", que dá nome ao presente trabalho, se configura para além dos processos analógicos com o chamado computacional design (Mitchel, 1985) que possibilita; um novo entendimento sobre a geração da forma; o processamento de dados gerados na modelagem 3D; e a sua fabricação. O desenvolvimento deste projeto através dos processos digitais permitiu desenvolver dois sistemas construtivo considerando diferentes opções dentro das escalas de pré-produção e pós-produção, o que permite a constante atualização do projeto. Esta integração pretende ultrapassar a concepção isolada da geometria em que o projeto é encarado de maneira sequencial. Estimula uma compreensão da integração das etapas da concepção, simulação e fabricação digital (CAAD-CAE-CAM), num processo assincrônico de projeto. Esta metodologia é desenvolvida através da aplicação de um estudo de caso teórico-prático analisando os impactos e transformações das etapas de projeto. Expondo desde as estratégias de representação, até à materialização, em que utiliza processos de fabricação com modelos combinados de técnicas aditivas e técnicas subtrativas para o mapeamento e construção na escala 1:1. 


\section{GERAÇÃO ALGORÍTMICA E PARAMÉTRICA \\ INICIAÇÃO À MODELAÇÃO DE FORMAS COMPLEXAS}

O Projeto, como referido foi iniciado em MDA, explorado um conjunto de exercícios geométricos e conceituais em que são introduzidos princípios básicos do software de modelagem tridimensional, o Rhinoceros. Para testar os conhecimentos e se familiarizar com o programa os participantes começam por representar um vegetal, de geometria complexa em ambiente tridimensional. Exploram, assim, o raciocínio geométrico para gerar de diferentes maneiras a sua superfície.

Este vegetal começa por ser fotografado, sendo a informação introduzida, no Rhinoceros, nas quatro vistas. Depois é pedido que utilizando os comandos de geração de superfícies ensinados seja recriado o vegetal, utilizando o mínimo de informação possível. É assim encorajada a geração com poucos elementos, em detrimento de uma geração foto-realista, com muitos pontos, mas que geram geometrias pouco operativas e de difícil modificação. É estimulada a utilização de curvas NURBS de maneira eficaz e consciente.
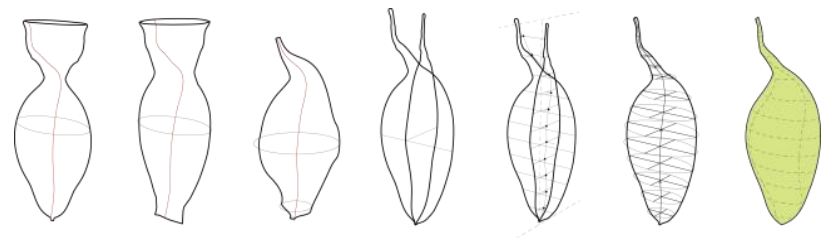

Figura 1. Testes de aproximação do vegetal em ambiente tridimensional. Fonte: autores.

$\mathrm{Na}$ disciplina são realizados alguns exercícios, que partem do vegetal representado - que deve ser coerente geometricamente, formando uma superfície fechada (closed Breap), com a realização de composições combinando esse vegetal, à operações booleanas e seções do fruto para formar um espaço protegido. O objetivo final do exercício é criar um abrigo com múltiplas funções, de forma livre e abstrata com base no objeto/vegetal de origem. Na disciplina estas operações são executadas em Rhinoceros para que os alunos se familiarizem com a modelagem, sendo a programação visual em grasshopper introduzida apenas num exercício seguinte (Componentes magnéticos). Neste caso, depois do exercício da disciplina a forma foi explorada utilizando programação visual .

\section{FORMA PRIMITIVA E COMPOSIÇÃO BOOLEANA}

O vegetal utilizado no desenvolvimento da forma primitiva foi o tamarindo (gênero botânico Tamarindus) que neste caso foi combinado, criando uma composição com 6 elementos que interagem entre si, criando um volume único. Nesta composição o resultado é alterado variando o volume, posição e rotação de cada elemento da composição em diferentes interações. É depois criada outra composição utilizando operações booleanas de adição e interseção para criar o espaço do abrigo. O espaço interior/exterior fluido e orgânico, se destaca do padrão convencional que separa parede, chão e teto; dando lugar a áreas fluídas com superfícies contínuas.

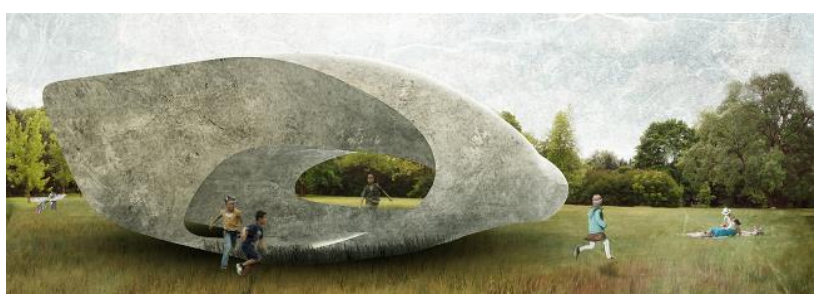

Figura 2. "Abrigo de forma livre" gerado através de composições booleanas. Fonte: autores.

A pesquisa que será apresentada a seguir traduz o aprofundamento dos processos generativos e do raciocínio geométrico, iniciados na referida disciplina. A partir dessa etapa o trabalho passa a ser desenvolvido individualmente como trabalho final de conclusão de curso e através da programação visual, explorando de maneira mais abrangente o campo de processos CAADCAE-CAM.

\section{PROGRAMAÇÃO VISUAL}

No aprofundamento do estudo dos processos generativos foi necessário que a geometria, gerada em Rhinoceros, fosse interpretada novamente no Grasshopper. O objetivo foi entender os limites formais das transformações, principalmente, quanto ao tipo de modelos capazes de atender às múltiplas funções e compreendessem ideais de continuidade espacial geométrico entre espaços internos e externos. Esse entendimento entre intenção e resposta do projeto, define uma proposição para a criação de parâmetros.

Para alterar a composição booleana que gerou o primeiro abrigo foram aplicadas transformações isométricas de: volume, posição e rotação. Logo, no desenvolvimento do sistema algorítmico cada um desses parâmetros foi trabalhado separadamente para se compreender os limites das transformações e estudar a geometria resultante. Os elementos que sofreriam deformação, ou seja, a que foram aplicadas as transformações variando parâmetros, foram 4 vegetais posicionados nos limites externos dos outros 2 vegetais centrais. Estes vegetais centrais não sofreriam nenhuma transformação isométrica, apenas a operação booleana, de subtração ou adição.

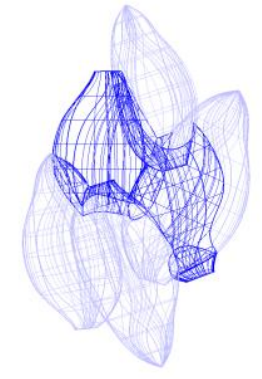

Figura 3. Relações de interação entre os elementosvegetais. Fonte: autores.

As operações booleanas acima descritas tiveram como intuito gerar diferentes soluções. As geometrias resultantes descreveram a existência de um, ou mais, domínios numéricos para cada transformação isométrica. A avaliação sobre a continuidade e capacidade formal de ocupação definiu, o que foi designado como domínio funcional da geometria final. 
"In a greater relevance to design and the designer, the most general application of computation is in producing outcomes realized from the processing of internal and external properties. Computation provides a framework for negotiating and influencing the interrelation of these datasets of information, with the capacity to generate complex order, form and structure." (Menges \& Ahlquist, 2011,p.13)

Na citação acima, Menges e Ahlquist afirmam que a lista de dados numéricos gerados na modelagem permite que se tenha uma resposta na geometria, ou na estrutura de dados do modelo. Em uma visão simplificada, se trata de utilizar a computação no controle da intenção de projeto e de visualizar esse controle na resposta do projeto simultaneamente. A computação, nesse caso, é como uma ponte facilitadora ou uma ferramenta que estreita essas duas relações. Nesse sentido, entendemos que é possível antecipar a visualização do resultado final, ou, antecipar inúmeros resultados finais. Nessa antecipação, surge a possibilidade de se controlar as informações que se pretende, ou seja, atuar apenas naquelas que respondem a uma intenção de projeto.

Partimos então, para uma visão de criar critérios de seleção desses dados. $\mathrm{Na}$ pesquisa em questão, demonstramos que o domínio numérico encontrado para cada transformação isométrica deve responder a dois aspectos formais do projeto; os limites das superfícies que permitem gerar uma espacialidade contínua e a relação entre massa construída e os vazios internos.

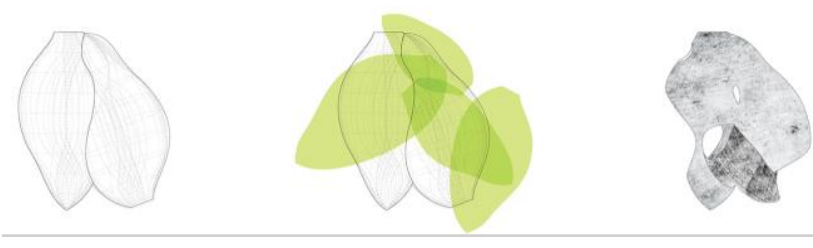

Figura 4. Exemplo da atuação dos parâmetros de rotação. Fonte: autores.

Ainda na citação, a questão sobre como a estrutura de dados pode ser usada para manipular modelos cada vez mais complexos, é visto nesse momento da presente pesquisa para, gerar múltiplas opções formais, traduzidas na geração de uma iamíiia de soiuções espaciaimente possíveis. Veremos que na fabricação essa estruturação dos dados vai interferir no manejo dos dados para sua execução e, consequentemente na complexificação do modelo. Vimos assim que, a maneira como um sistema algorítmico se estrutura pode definir um conjunto de respostas eficientes da intenção de projeto ou apenas um conjunto de respostas. Na seleção abaixo, as três geometrias para cada parâmetro de transformação representam, graficamente, o conjunto de possibilidades que o sistema algorítmico gerou e as respostas formais que atendem aos critérios definidos.

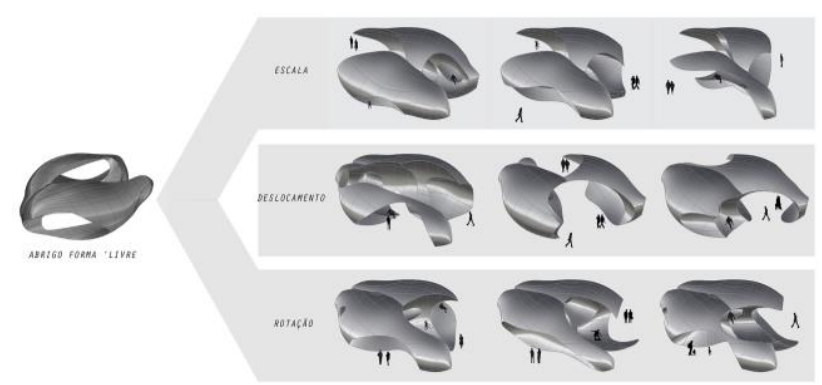

Figura 5. Árvore-família de soluções. Fonte: autores.
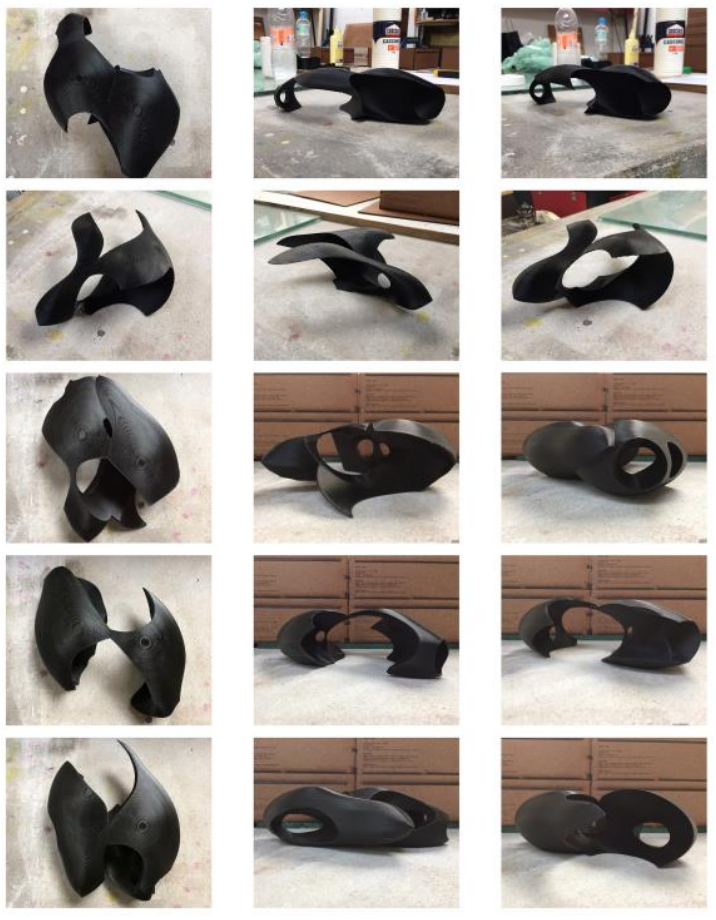

Figura 6. Modelos fabricados em escala reduzida. Fonte: autores.

Para concluir a compreensão dessa etapa, as geometrias então, foram interpretadas e inseridas em diferentes contextos urbanos da cidade afim de aproximar a leitura desse objeto com a realidade. Os cenários escolhidos acentuam a versatilidade formal e urbana dos objetos criados relativamente ao contexto em que são inseridos, sem objetivar ou limitar a sua função, respondendo a problemáticas urbanas locais. Os objetos podem ser entendidos como elementos escultóricos de uso livre capazes de reconstruir cenários da cidade através de novas perspectivas. 


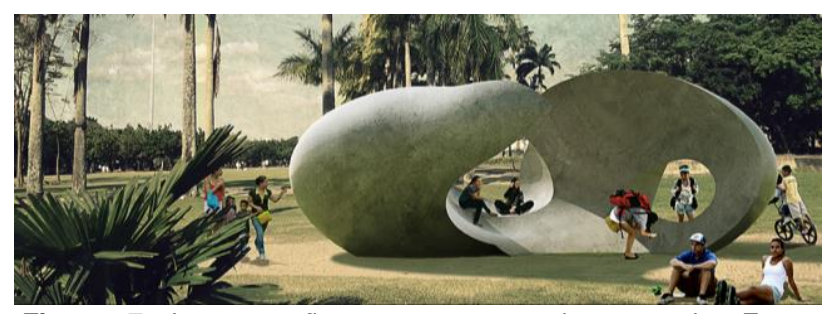

Figura 7. Interpretação em contexto urbano-escala. Fonte: autores.

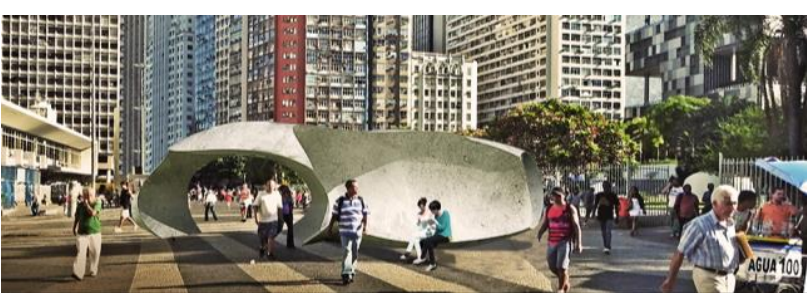

Figura 8. Interpretação em contexto urbano-direção. Fonte: autores.

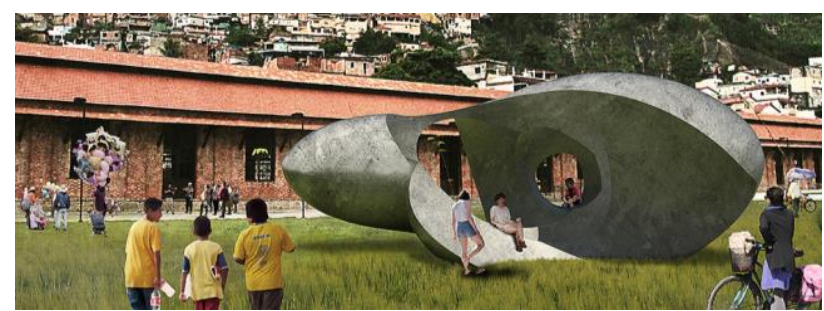

Figura 9. Interpretação em contexto urbano-rotação. Fonte: autores.

\section{FABRICAÇÃO DIGITAL ESTUDO DE CASO}

Esta etapa representou a aplicação prática-construtiva da pesquisa. Para isso, foi selecionada uma geometria da família de soluções para ser o estudo de caso. Iwamoto (2009) no seu livro Digital Fabrication apresenta 5 técnicas de fabricação; Sectioning, Tessellating, Folding, Contouring e Forming. Foram desenvolvidas duas técnicas construtivas a de contouring, que traduzimos como desbaste 3D; e a de sectioning, que traduzimos como planificação 2D. Estas técnicas utilizaram como materiais, respetivamente, blocos de EPS (poliestireno expandido) com uma fina camada de revestimento em concreto compósito; e a fabricação bidimensional de chapas de compensado perfuradas, num processo de desbaste 2D fixadas com cabos de aço. A utilização destas duas técnicas aplicadas à mesma geometria permitiu uma análise comparativa entre os processos e uma compreensão mais profunda do que significa processo integrado e o design computacional no desenvolvimento de projeto.

\section{GEOMETRIA CONSTRUTIVA I : DESBASTE 3D}

Como referência para a técnica de subtração por desbaste de poliestireno expandido (EPS), utilizando uma CNC Router de 5 eixos, foram estudados dois projetos de superfície contínua.

O primeiro caso estudado, foi o projeto de uma estação de ônibus The Amazing Whale Jaw, do escritório NIO Architecten, construído na Holanda em 2003 (Henriques 2015). Todo em superfície contínua autoportantes, o projeto não utiliza elementos de transição, como pilares estruturais, não havendo a separação entre elementos horizontais e verticais. Para realizar tal construção foram

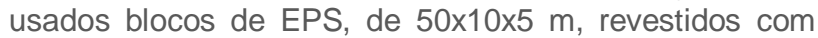
poliéster. A técnica é semelhante à utilizada na fabricação das pranchas de surf, com desbaste manual. Uma das vantagens dessa técnica é a de diminuir drasticamente as cargas relativas do peso próprio dos materiais, compreendido apenas de 14 toneladas, e de o EPS funcionar apenas como preenchimento leve. O uso de uma fina camada de poliéster sobre os blocos, após colados, dá resistência estrutural ao objeto. A casca, sofre variações de espessura de acordo com o reforço estrutural que determinadas zonas da curvatura exigem. Estas variações são controladas basicamente pelos raios (inferiores a $100 \mathrm{~mm}$ ) das zonas de transição da superfície (curvas booleanas) limitando o excesso de tensões.

O outro caso estudado, foi o projeto da ponte Spencer Dock Bridge, do escritório AL_A, construída em Dublin entre 2006-2009. Sua geometria paramétrica foi remodelada em SolidWorks para criar blocos de EPS quadrados e, assim, garantir o controle da tolerância construtiva na variação da superfície. Os blocos, de $3 \times 1,25 \mathrm{~m}$, foram pulverizados com material impermeabilizante (polyurea) e lixados. Depois de montados ainda foram aplicadas camadas adicionais de concreto misturado com calcário e incorporados, espaçadores, de $10 \mathrm{~mm}$, entre os blocos para permitir variações de dilatação do material.

Estes casos foram analisados com a intenção de dar suporte a aplicação e adaptação da técnica de subtração (desbaste) ao estudo de caso aplicado. No mercado brasileiro o material EPS foi encontrado nas dimensões equivalentes a $4 \times 1,2 \times 1 \mathrm{~m}$. A partir das dimensões do bloco, a geometria foi interpretada como se tivesse que ser dividida em partes de $4 \times 1,2 \times 1 \mathrm{~m}$. Ou seja, era necessário fazer uma interseção entre a geometria com o conjunto de blocos de EPS. Com esse intuito, foi concebida uma grid em 3 eixos ( $X, Y$ e $Z$ ), em que 0 distanciamento entre os planos seriados seria de $4 \times 1,2 \times 1 \mathrm{~m}$. Um aspecto é compreender que a utilização do bloco unitário como unidade, micro, quando multiplicada, para atingir o macro, garante uma noção de escala e proporção real ao objeto. Além deste aspecto, podemos observar que o módulo valerá como unidade de uso comparativo, deixando de ser tão necessário compreender a variação volumétrica dos conjuntos e fazendo valer o quantitativo de unidades de blocos. A geometria, nesse momento, começa a sofrer a primeira avaliação construtiva ainda que sem sofrer deformações e ações diretas em sua forma. Utilizando a interseção, poderíamos explorar a variação da maior face do bloco, de $4 \mathrm{~m}$, nos 3 eixos e, consequentemente, avaliar numericamente e fisicamente o comportamento da interseção booleana. Ou seja, que variação assegura 0 menor número de blocos e o melhor aproveitamento dos blocos. Blocos melhor aproveitados são aqueles com desbastes em menos faces e que, em volumetria perdem menos $\mathrm{m}^{3}$. Em um primeiro momento, esta avaliação se fez por comparação, entre o número de blocos que fazem interseção com a grid e os que não fazem, e também entre os comportamentos, dos 3 eixos em relação a forma global. 

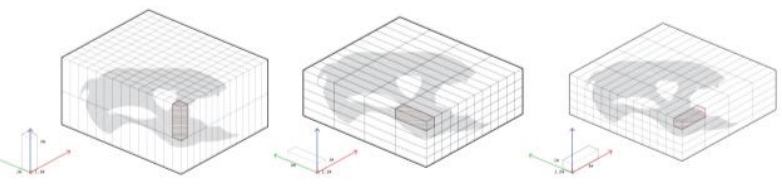

Figura 10. Variações do bloco nos eixos Z,Y e X. Fonte: autores

Com a interseção, a variação em X gerou um total de 202 blocos e a fragmentação da geometria global em unidades menores, permitiu viabilizar a fabricação. Para o efeito foi feita a classificação de unidades a partir da visualização das superfícies de desbaste e, considerando o maquinário, CNC Router de 5 eixos, as superfícies foram classificadas em 3 tipos: superfícies côncavas, superfícies convexas, e superfícies côncavas e (+) convexas. Como resultado dessa primeira classificação; 68 casos de superfícies côncavas, 57 convexas e 78 côncavos e (+) convexos.

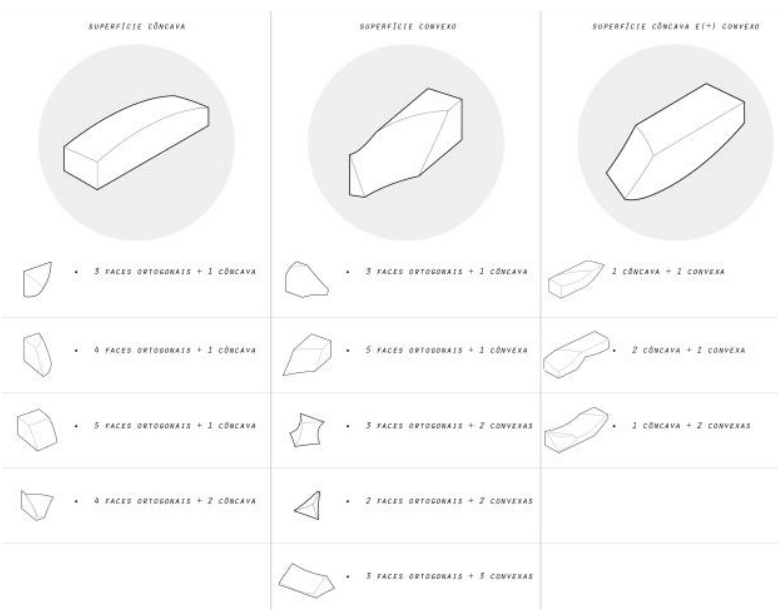

Figura 11. Mapa da classificação em tipos e subtipos. Fonte: autores.

Esse quantitativo, permitiu a criação de subtipos classificados pelo número de faces; ortogonais, côncavas, convexas e côncavas $\mathrm{e}(+)$ convexas. Os blocos, então, foram agrupados detalhadamente, analisados e foram definidas estratégias de desbaste para todos os casos. Nas situações mais desfavoráveis, dois casos, a interseção fragmentou tanto a forma que suprimiu os encontros das arestas ortogonais, evidenciando a fragilidade estrutural da geometria nesses pontos.

Na execução do modelo, em escala, com impressão 3D, foi feita uma série de simulações globais que confirmavam a hipótese da avaliação dos raios de curvatura corretos, observada depois da separação dos blocos em subtipos. No modelo em escala 1:50, a geometria global foi fatiada em 5 grandes peças em que, cada uma, utilizava quase a totalidade da área da máquina, sendo fabricadas separadamente. Assim obtivemos um modelo, que ocupava uma área de $20 \times 20 \times 6 \mathrm{~cm}$. A divisão, em 5 peças, foi feita a partir da grid de blocos de EPS e permitiu a otimização da execução da forma sem o uso de suportes. Para tanto, foi trabalhado o reposicionamento das peças em diferentes orientações. Sem os suportes, otimizamos o uso do material, o tempo de máquina e, a construção, aditiva, das curvaturas internas sem falhas.
A fabricação permitiu observar determinados pontos da geometria aonde a junção entre 2 raios de curvatura era tão fina (sem espessura) que a máquina, não fazia a adição da camada de filamento. A correção gerada pelo g-code do Simplify foi recriar essas 2 curvaturas para execução a geometria em máquina. $O$ resultado fabricado apresentou pequenas falhas nas curvaturas quando próximos das junções e fissuras entre as camadas de filamento. Para corrigir, retornamos ao Grasshopper, para a variação do parâmetro de transformação afim de controlar a espessura nessas junções. Os códigos numéricos demonstraram dificuldade em executar algumas transições por causa da tolerância da máquina aos raios de curvatura do modelo. Por mais que fossem coerentes matematicamente, para execução 'mecânica', não demonstraram coerência no design da geometria.

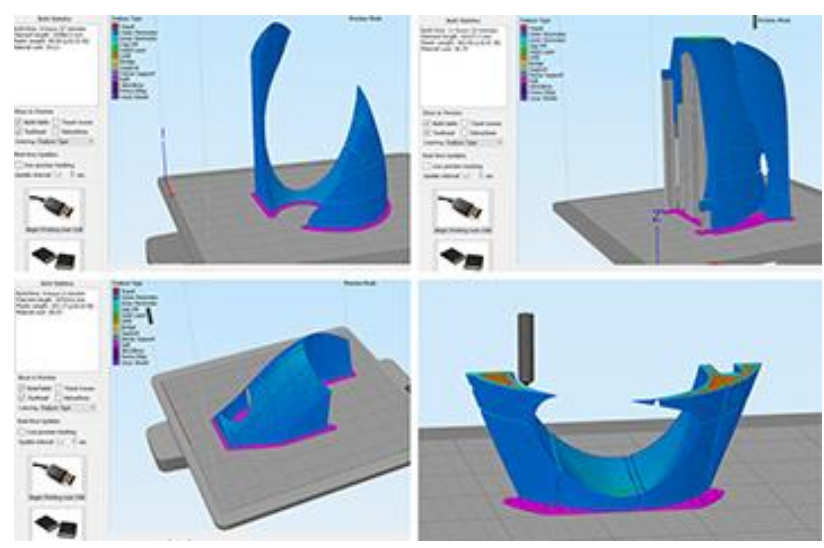

Figura 12. Análises para fabricação em impressão 3D. Fonte: autores.

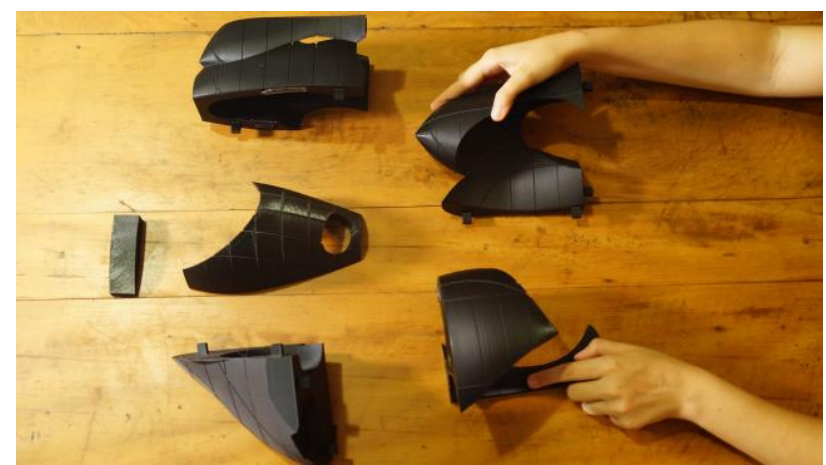

Figura 13. Modelo fatiado em 5 partes executado em 3D printer. Fonte: autores.

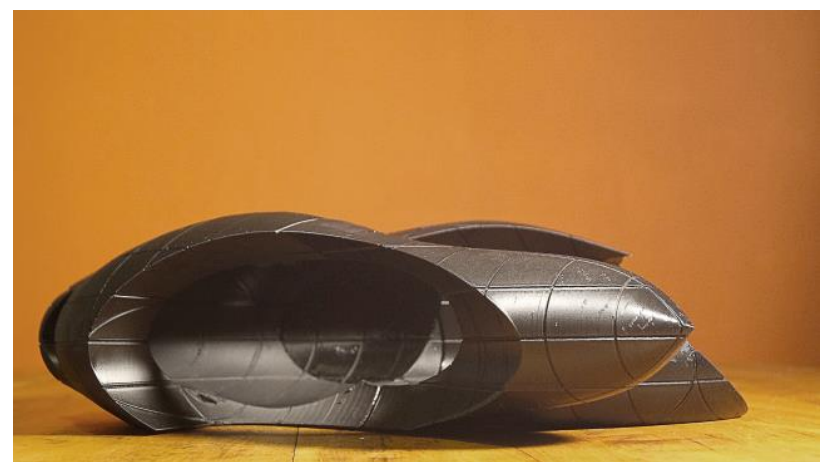

Figura 14. Modelo montado em escala 1:50. Fonte: autores.

Enquanto na fabricação em escala não houve necessidade de desenvolver uma organização de montagem, na fabricação 1:1 isso seria diferente. A título de teste, foi desenvolvido uma ordenação lógica para nomenclatura das unidades de EPS. A partir, da grid dos 
planos de fatiamento, criada nos eixos $X, Y$ e $Z$ e nas medidas da unidade, a grid numérica e alfabética constituiu a ordenação no eixo $X$ (número total linhas $\mathrm{X}$ ), depois colunas alfabética em $Y$ (n em y) e novamente numérica em $Z$ ( $n \mathrm{z}$ ). Logo compreende-se, que em $X$ vamos até o 35, em $Y$ ate I e em $Z$ ate 4 . O processo de montagem foi organizado pelo empilhamento em $Z$.

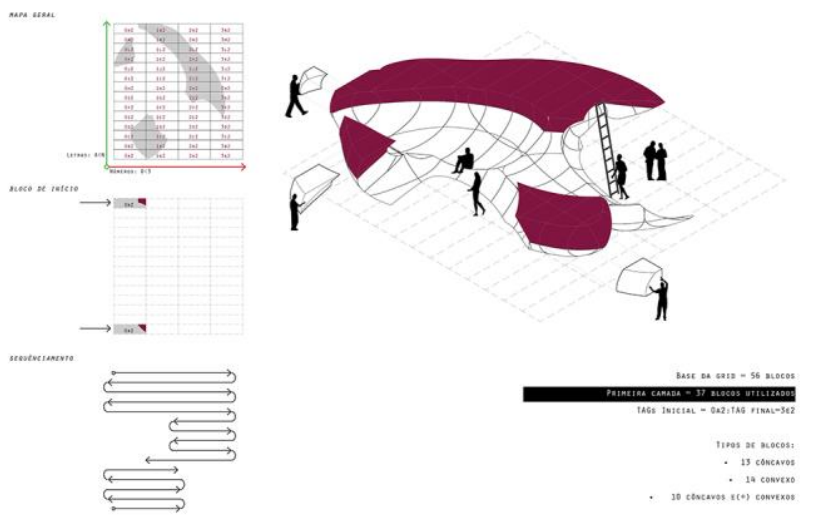

Figura 15. Processo de montagem ilustrado-resumido. Fonte: autores.

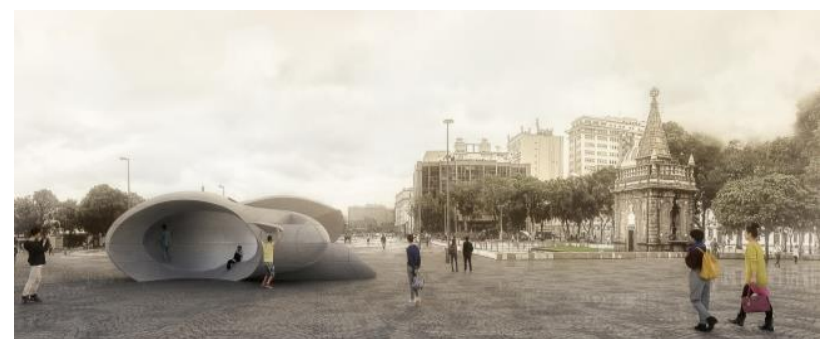

Figura 16. Imagem renderizada e contextualizada da técnicamontagem. Fonte: autores.

\section{GEOMETRIA CONSTRUTIVA II: CORTE BIDIMENSIONAL}

$\mathrm{Na}$ geometria construtiva II, foi aplicada a técnica de planificação, por cortes 2D, em chapas de compensado perfuradas e cabos de aço. Durante o processo de modelagem, por programação visual, houve inúmeros debates inconclusos que só foram saciados depois da montagem do modelo físico, em escala. Analiticamente, a técnica de 'sectioning' exigiu um diálogo mais intenso entre os processos CAAD-CAE-CAM justificando a pesquisa no âmbito da experimentação e reforçando a afirmação da interação ativa entre os processos computacionais.

Devido à técnica ser amplamente conhecida, as dificuldades em a realizar se encontram na questão de compatibilizar a geometria, técnica e escala das partes. Os projetos analisados como referencia, acatam separadamente algumas questões que encontramos durante o processo, mas nenhum dos dois trabalha as três questões simultaneamente. Assim, os exemplos serão brevemente citados, mas não descritos. No projeto de John Bridge, em Dublin, em 2003, a regularidade formal da superfície oval facilitou a planificação do modelo, nos eixos $\mathrm{X}$ e $\mathrm{Y}$. No segundo exemplo, o pavilhão de verão desenvolvido por Brakow Leibinger, para o Museu Alemão de Arquitetura, em Frankfurt a geração da forma foi explorada a partir da curva de Moebius e, os modelos em escala planificados apenas em um eixo resolvem as interseções entre curvaturas. Para o pavilhão 1:1, o corte em uma seção que não tem interseção entre curvaturas de raios diferentes permitiu trabalhar a planificação em 2 eixos, $\mathrm{X}$ e $\mathrm{Y}$, e fazer $\mathrm{O}$ travamento da curvatura.

$\mathrm{Na}$ geometria construtiva II, quando dividida em planos, orientados em $\mathrm{X}$ e seriados em $\mathrm{Y}$, as polylines criadas tiveram diferentes comportamentos. Ou seja, tínhamos polylines diferentes, todas fechadas, mas algumas compostas (path), outras de perfis complexos. Em um primeiro momento, isso foi resolvido por agrupamentos por semelhança. Para alguns casos mais específicos foi feito o redesenho das polylines, separadamente, com o objetivo de corrigir alguns erros manualmente. Mas as imprevisibilidades obtidas das interseções booleanas foram se perdendo gradualmente. Então, o 'rebuild' manual foi excluído como solução e, a opção foi avaliar o número de planos gerados afim de manter a coerência dos espaços internos e externos da geometria. No caso das polylines complexas, algumas regiões do seu comprimento configuravam formas que dificultavam o mapeamento dos furos percorrendo o perímetro das chapas. O que obrigou a mapear os furos em toda a chapa, ou seja, com uma grid. Essa grid representava os cabos de aço e, foi orientada em $Y$ e seriada em $X$, que intersectava as superfícies das chapas gerando os furos. Como resultante formal, ocorreu a supressão e simplificação das cavidades internas do modelo que só foi compreendida totalmente pós fabricação.

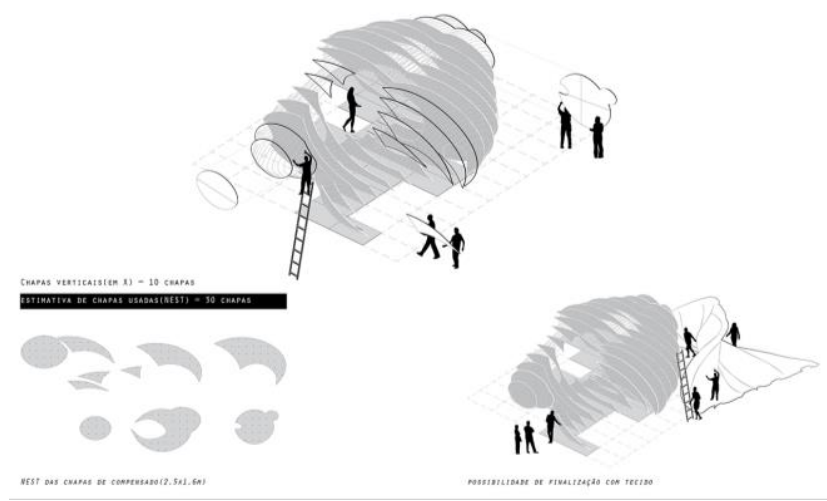

Figura 17. Processo de montagem ilustrado-resumido. Fonte: autores.

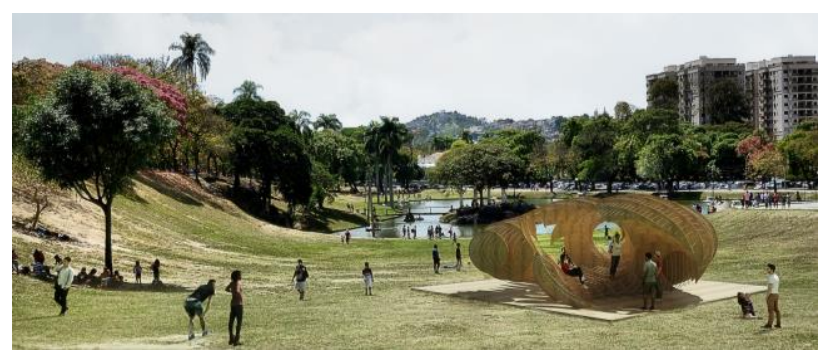

Figura 18. Imagem renderizada e contextualizada da técnicamontagem. Fonte: autores.

\section{RESULTADOS}

A fabricação dos modelos, em escala 1:50, em diferentes técnicas e materiais, permitiu fazermos direcionamentos específicos das estratégias de projeto para uma possível construção 1:1. 
Na geometria construtiva I a classificação dos blocos; a ordenação de montagem; e a leitura numérica do g-code, foram os principais pontos de análise. No caso da classificação, o agrupamento em subtipos sugere uma avaliação sobre o valor dos raios de curvatura gerados nas unidades dos blocos. Fica em aberto, se seria mais lógico definir tolerâncias sobre o grau dos raios na geração da geometria global, ou seja, pré-operações booleanas. Ou se, essa tolerância deve acontecer pósoperações booleanas quando já se configuram como blocos a serem desbastados. Na ordenação das peças, o mapeamento lógico sugere uma revisão, em que o ponto de partida de montagem deva começar no eixo $Z$ e não no eixo $X$. Na fabricação, o que se questiona é a leitura das superfícies NURBS para meshes. A necessidade em equilibrar as espessuras das junções a adição de um número mínimo de camadas de filamento para leitura numérica em Simplify3D configurou uma fabricação assistida. O que trouxe questionamentos quanto como seria mais eficiente trabalhar, na manipulação dos dados, em Grasshopper; na manipulação do número de pontos para gerar as meshes ou; em trabalhar toda a programação visual em mesh.

Na geometria construtiva II, a estruturação da árvore de dados do algoritmo, e o jogo de equilíbrios entre os dimensionamentos, distâncias e materialidade do conjunto mapeou os principais pontos de análise. A estruturação da árvore de dados, quando executamos a planificação da geometria, gerou uma complexidade entre a interseção das polylines, em $X$ e $Y$, que os agrupamentos feitos não demonstraram eficiência para uma execução em 1:1. Uma estratégia, seria tentar atuar globalmente, e gerenciar paralelamente os dados de $\mathrm{X}$ e Y. Já nas relações entre as partes, dimensionamentos e materialidade, a tensão criada pelos cabos de aço enrijeceu muito conjunto. Tanto a posição e relação entre os furos, para os cabos passarem, quanto o distanciamento e espessura das chapas (no caso, acrílico) são fatores de manipulação no modelo. Essa tensão poderia ser simulada e avaliada posteriormente através de um plugin em Grasshopper.

A possibilidade de se fabricar o mesmo modelo em técnicas e materialidades diferentes permitiu 0 aprofundamento da apreensão dessa geometria. Quando trabalhamos sua segmentação em blocos tridimensionais compreendemos suas curvaturas como cascas a serem desbastadas e, observamos as curvaturas de suas superfícies, parte por parte. Quando segmentamos o modelo em chapas bidimensionais observamos o modelo globalmente, pelo seu perímetro segmentado, não conseguimos ter uma atuação tão detalhada e local nas curvas. A conclusão desse comparativo não se pauta em escolher qual a melhor técnica em converter uma geometria complexa em geometria construída, mas sim em qual técnica permite se aproximar profundamente dos pormenores da geometria. Se uma técnica, permite avanços contínuos na simplificação, ou visualização em detalhe, de sua complexidade significa que progressivamente o designer está desvendando aquela forma. O desvendar, nos dá a possibilidade de definir estratégias, otimizações e eficiências, seja no âmbito da construção seja no da modelagem digital.

"Fundamental to computational design is the understanding of how systems, as form and as mathematical ordering constructs, operate. Fundamental to understanding their operation is the level of prediction contained in the model, which envisions the system. Initially, this was a primary consideration for the first graphical systems in architecture, stated as: 'With the help of computer-aided models, the designer will be able to predict the performance of any design alternative he may generate'. The success of this depends upon design paradigms which are oriented towards defining systems with concise parameters, and using the effort of prediction and feedback to inform, further specify and define overall the behavior of the organized system." (Menges \& Ahlquist, 2011,p.16)

Através da citação, acima, podemos fazer uma reflexão entre o 'desvendar' a geometria através do uso da computação e, a visão técnica de Menges e Ahlquist sobre geração de sistemas e os paradigmas do design. Quando os autores afirmam que para o designer fazer um melhor aproveitamento do uso da computação ele precisa prever, ou seja, se antecipar na ordenação de sistema matemático conciso que gere uma/varias respostas que atendam aos paradigmas de design definido pelo designer. Isso quer dizer que, é necessário ser eficaz no momento de definir uma primeira estratégia de codificação da geometria. Criar um sistema conciso e matematicamente eficaz significa possibilitar os avanços em apreende-lo totalmente e, consequentemente, apreender a forma que está sendo gerada através dele. $\mathrm{O}$ resultado dessa relação, de design e o designer é a coerência entre intenção e resposta do projeto. Nesse sentido, é possível avaliar que na técnica da geometria construtiva I o sistema criado permite estabelecer essa relação de intenção e resposta de projeto. A seguir poderemos ampliar essa discussão associando a geração da forma a sugestão da fabricação pré-concebida.

\section{DISCUSSÃO}

Ao longo do artigo foi utilizado o termo Geometria Construtiva associado a dois momentos do projeto arquitetônico. Um envolvendo a geração algorítmica e, outro relacionado com os processos de fabricação do objeto. Neste sentido o momento de geração foi anterior ao processo de fabricação, isto é, produzimos o modelo sem definir os materiais que iríamos utilizar, nem as técnicas que aplicaríamos. Ao nível de projeto utilizamos os recursos de modelação digital fundamentados em geração da forma, nomeadamente algoritmos paramétricos e programação visual (Oxman, 2006). Uma vez definido o modelo ao nível de fabricação utilizamos duas de cinco técnicas categorizadas (Iwamoto, 2009), no caso "Sectioning" e "Contouring".

Fica claro que poderíamos ter representado a fabricação por qualquer uma destas cinco técnicas. Contudo a representação formal obtida entre os processos de fabricação e os materiais utilizados ofereceu resultados diferenciados. Sendo assim um ponto de discussão surge a partir da relação entre o modelo e a fabricação, os seus materiais e as técnicas de fabricação. No caso a diferença entre "Desbaste 3d" e "Corte bidimensional" (Contouring e Sectioning), torna evidente a diferenciação entre massa e plano entre sólido absoluto e um sólido descontruído entre cheios e vazios.

Essa idéia, geometria construtiva, também pode ser pensada recorrendo ao estudo do construtivismo russo, um movimento artístico do início do séc. $X X$, que reivindica um novo papel transformador social da arte. Propõe redefinir o vocabulário artístico do historicismo, 
estabelecendo um vocabulário baseado em operações codificadas com elementos geométricos e cores primárias. Tendo surgido após o cubismo, o construtivismo influenciou diretamente as vanguardas artísticas européias como o neoplasticismo, o De Stijl, ou a Bauhaus.

O construtivismo russo adquire, segundo alguns historiadores (Jencks, 2006), dois significados diferentes. Se por um lado valoriza o papel abstrato da arte imaterial, por outro vaticina a utilização de novas tecnologias e materiais como o ferro e o aço, e mais tarde o concreto. Enquanto a primeira conotação está mais associada ao legado expressivo de Malevitch, Kandinsky, a segunda está mais associada com Tatlin ou Shukhov e com o desenvolvimento da técnica em determinadas obras arquitetônicas. No nosso caso a geometria construtiva também adquire os dois significados através de operações de transformação algorítmica da forma e, pela procura construtiva de processos de fabricação digital. Neste caso pode se discutir qual deverá vir primeiro a forma, ou a técnica (de fabricação), uma discussão clássica de projeto. O que realmente muda no processo integrado digital, é que estes processos apesar de não serem simultâneos podem ter uma interação constante e, o material e processos podem mudar a geometria e viceversa. Esta conclusão vem reforçar a necessidade de repensar o processo linear tradicional utilizando os processos computacionais para expandir o universo de soluções, desenvolvendo uma família de soluções, geométricas e construtivas, aplicadas e testadas em um projeto específico, como neste caso apresentado.

\section{AGRADECIMENTOS}

Gostaríamos de agradecer o suporte do LAMO Laboratório de Modelos 3D e Fabicação Digital e a toda a equipe; em especial ao Thiers Freire pela assistência nas primeiras impressões 3D; ao PROURB - Programa de Pós-Graduação em Urbanismo pelo apoio; à Márcia Anaf Wagner do Maths and Arts Fabrication - Manaf3D - pelas impressões 3D finais e análises do modelo; a assistência técnica de Affonso Orciuoli e Mauricio Matias da Rede Brasileira de Fabricação Digital; as ilustrações realistas de inserção urbana de Fernando Bonini. Este projeto foi premiado com uma menção honrosa em 2017 no
Concurso Arquiteto do Amanhã atribuído pelo Instituto dos Arquitetos do Brasil - IAB-RJ.

\section{REFERÊNCIAS}

Duhn, Nick. (2012). Digital Fabrication in Architecture. Laurence King Publishing Ltd, 41, 60-62, 98, 134-135.

Duarte,J; Celani, G.; Pupo R. (2011), "Inserting computational technologies in architectural curricula", em Gu, Ning andWang,Xiangyu(ed.), computational Design Methods an d Technologies: Applications in CAD, CAM and CAE Education, Hershey, PA: IGI Global.

Iwamoto, L. (2009). Digital Fabrication: Architectural and Techniques. Nova Iorque, NY: Princeton Architectural Press

Jenks, C. (1985). Modern Movements in Architecture. Lisboa, PT: Edição 70

Kolarevic, B. (2003). Designing and Manufacturing Architecture in the Digital Age. Londres, UK: Spon Press, Taylor \& Francis Group

Henriques, G. (2016), "Arquitetura algorítmica: Técnicas, processos e fundamentos", Anais IV ENANPARQ, Sessão 39. Projeto digital e fabricação na arquitetura: ensino, pesquisa e desafio, Organização: Cláudia Costa Cabral, Carlos Eduardo Comas, edição PROPAR/UFRGS, Porto Alegre.Disponível

www.researchgate.net/publication/305827549

Henriques, G.; Passaro, A.; Nobrega, A. (2017) "Tentáculos: recriando criaturas híbridas, analógico-digitais". XXI Congreso de la Sociedad Iberoamericana de Gráfica Digital, 2017, Concepción Chile. Editora Blucher, S Paulo, pp. 530-7, DOI: 10.5151/sigradi2017-082 ISSN 23186968.www.researchgate.net/publication/321232704

Henriques, G. (2005). "Novos processos de construção". Revista Arquitectura \& Vida, $\mathrm{n} \div 58,100-106$. Disponível em www.researchgate.net/publication/274387816

Mitchell, W. J. (2008). A lógica da Arquitetura: Projeto computação e cognição. Campinas, BR: Editora UNICAMP

Terzidis, K. (2003). Expressive Form: A conceptual approach to computational design. Londres, UK: Spon Press, Taylor \& Francis Group

Menges, A. \& Ahlquist, S. (2011). Introduction: Computacional Design Thinking. In A. Menges \& S. Ahlquist (Eds), AD Reader: Computacional Design Thinking (pp.10-21). Londres, UK: John Wiley \& Sons Inc.

Oxman, R. \& Oxman, R. (2014). From Composition to Generation. In R. Oxman \& R. Oxman (Eds.), Theories of the Digital in Architecture (pp. 55-61). Londres, UK: Routled 